\title{
Structure and properties of edge dislocations in $\mathrm{BiFeO}_{3}$
}

\author{
Piyush Agrawal, ${ }^{1,2,{ }^{*}}$ Marco Campanini, ${ }^{1, *}$ Andrew Rappe,${ }^{3}$ Shi Liu, ${ }^{3}$ Vincenzo Grillo, ${ }^{4}$ Cécile Hébert, ${ }^{5}$ \\ Rolf Erni, ${ }^{1}$ Daniele Passerone, ${ }^{2}$ and Marta D. Rossell ${ }^{1, \dagger}$ \\ ${ }^{1}$ Electron Microscopy Center, Empa, Swiss Federal Laboratories for Materials Science and Technology, 8600 Dübendorf, Switzerland \\ ${ }^{2}$ nanotech@surfaces Laboratory, Empa, Swiss Federal Laboratories for Materials Science and Technology, 8600 Dübendorf, Switzerland \\ ${ }^{3}$ Department of Chemistry, University of Pennsylvania, Philadelphia, Pennsylvania 19104-6323, USA \\ ${ }^{4}$ CNR-Istituto Nanoscienze, Centro S3, 41125 Modena, Italy \\ and CNR-IMEM, Parco Area delle Scienze 37/A, 43010 Parma, Italy \\ ${ }^{5}$ LSME - Institut de Physique, École Polytechnique Fédérale de Lausanne, 1015 Lausanne, Switzerland
}

(Received 24 January 2019; published 28 March 2019)

\begin{abstract}
Edge dislocations are frequently found in epitaxial $\mathrm{BiFeO}_{3}$ multiferroic thin films and are expected to exhibit distinctive and localized magnetoelectric properties. However, an exhaustive characterization of these dislocations at the atomic level has to date been largely overlooked. Here, we use a combination of scanning transmission electron microscopy techniques, atomistic simulations obtained from classical molecular dynamics calculations, and real-space multiple-scattering theory to explore the chemical properties and the bonding characteristics of the atoms located at and near the dislocation cores. We find that in addition to Bi, small amounts of $\mathrm{Fe}$ atoms are present in the $\mathrm{BiFeO}_{3}$ dislocation cores which result in uncompensated $\mathrm{Fe}$ spins along the dislocations and give rise to a magnetic signal. Our results suggest that edge dislocations in $\mathrm{BiFeO}_{3}$ films could be efficiently used for realizing $\mathrm{BiFeO}_{3}$-based magnetic devices.
\end{abstract}

DOI: 10.1103/PhysRevMaterials.3.034410

\section{INTRODUCTION}

The ability of multiferroic materials to couple magnetic and ferroelectric orders continues to attract considerable attention because of their potential application in spintronics and memory devices [1-4]. Among them, $\mathrm{BiFeO}_{3}$ (BFO) is widely investigated because of its magnetoelectric properties at room temperature, with antiferromagnetic and ferroelectric transition temperatures $T_{\mathrm{N}} \sim 643 \mathrm{~K}$ and $T_{\mathrm{C}} \sim 1103 \mathrm{~K}$, respectively [5]. The bulk BFO phase displays a rhombohedrally distorted perovskite structure (space group $R 3 c$ ) [6,7] exhibiting a large spontaneous polarization of $\sim 100 \mu \mathrm{C} / \mathrm{cm}^{2}$ along the [111] direction of the perovskite cell [8,9] and G-type antiferromagnetic (AFM) order [10]; that is, ferromagnetic coupling of the spins within the pseudocubic (111) planes and antiferromagnetic coupling between the different planes. Despite the AFM ordering, the symmetry of the $R 3 c$ structure permits the establishment of a weak ferromagnetism due to a small spin canting originated by a Dzyaloshinskii-Moriya (DM) interaction of the antiferromagnetic sublattice [11-13]. Although the DM coefficient is one order of magnitude smaller than the superexchange interaction, the arising net magnetization due to canted antiferromagnetism makes BFO potentially exploitable for innovative spintronic/magnetoelectric devices working at room temperature [14-17]. However, a key issue impeding the use of BFO for magnetoelectric applications is the presence of a cycloidal spin modulation which is incommensurate with the crystal lattice (period $\sim 630 \AA$ ) $[18,19]$. This spiral modulation

\footnotetext{
*These authors contributed equally to this work.

†Corresponding author: marta.rossell@empa.ch
}

suppresses the net macroscopic magnetization arising from the canted spins and hinders the magnetoelectric coupling. The cycloidal modulation represents therefore an undesirable effect that narrows down the functional properties of $\mathrm{BFO}$ to the solely ferroelectric-related ones. In order to overcome this major limitation, extensive works have been addressed to the search of mechanisms able to destroy the cycloidal spiral ordering.

Among various proposed strategies to enhance the magnetoelectric coupling, chemical pressure by substitutional doping has been proven to induce a net weak magnetization in BFO [20-25]. Alternatively, strains arising in epitaxial thin films have also been explored to modify the magnetoelectric multiferroic properties of BFO by an appropriate choice of the substrate [15]. For example, a metastable polymorph of BFO with a strongly elongated unit cell (the so-called tetragonallike phase or $T$ phase) exhibiting a giant polarization as high as $150 \mu \mathrm{C} / \mathrm{cm}^{2}$ was stabilized under epitaxial strain in highly strained thin films grown on $\mathrm{LaAlO}_{3}$ substrates [26-29]. Conversely, relaxation of the misfit strain associated with the lattice mismatch between the BFO film and the substrate might also occur through the formation of various kinds of crystallographic defects, such as domain walls and dislocations [30-32]. Symmetry breaking at these positions can induce emergent behavior with properties that deviate significantly from the bulk. Thus, it is known that domain walls in multiferroics can manifest a net magnetic moment even if the domains themselves are antiferromagnetic or paramagnetic $[33,34]$. As such, domain walls in BFO have been extensively studied using both experimental and theoretical tools $[35,36]$. From a computational perspective, an atomistic potential based on the conservation principles of bond-valence 
sum (BVS) and bond-valence vector sum (BVVS) was developed to study phase transitions and temperature-dependent cation displacements in BFO [36] and domain-wall motions under applied bias [37].

Also, some studies have suggested the importance of dislocations on the magnetic properties of ferromagnetic [38,39] and antiferromagnetic [40] materials. One particularly interesting example was reported by Sugiyama et al. [40], where dislocation cores in $\mathrm{NiO}$ films grown on $\mathrm{SrTiO}_{3}(001)$ substrates were found to be nonstoichiometric with the help of electron energy loss spectroscopy (EELS) and density functional theory (DFT) calculations. They demonstrated that the detected Ni deficiency at the dislocation cores was the main cause for the ferromagnetic behavior of the otherwise antiferromagnetic $\mathrm{NiO}$.

Edge dislocations in BFO thin films were investigated by Lubk et al. [32] using spherical aberration-corrected scanning transmission electron microscopy (STEM). Employing a combination of model-based structure analysis and phase field simulations, they were able to evaluate the strain-polarization coupling in the presence of strong anisotropic strain. Besides, an atomic model for the dislocation core structure was derived in that study by comparing atomically resolved high-angle annular dark-field (HAADF) STEM and simulated images. However, possible deviations from stoichiometry at the core of the BFO edge dislocations in combination with theoretical modeling were not considered.

The aim of the present work is to deliver an atomistic model of the commonly observed edge dislocations in BFO films (with Burgers vector $\mathbf{b}=[001]$ and dislocation line $\xi=[100])$ and to derive a correlation between their structural, electronic, and magnetic properties. For this, we use atomic-resolution aberration-corrected HAADF-STEM combined with energy-dispersive X-ray (EDX) spectroscopy and EELS as well as off-axis electron holography. Several atomistic models of edge dislocations accommodating different $\mathrm{Bi} / \mathrm{Fe}$ concentrations are derived using molecular dynamics with bond-valence (BV) model potentials [36] for BFO, and are further benchmarked against the experimental data. Subsequently, the experimental EELS results are interpreted with the aid of simulations based on a real-space multiplescattering code [41].

\section{RESULTS AND DISCUSSION}

Samples consisting of either a $\mathrm{BiFeO}_{3} / \mathrm{La}_{0.7} \mathrm{Sr}_{0.3} \mathrm{MnO}_{3}$ (BFO/LSMO) or a $\mathrm{BiFeO}_{3} / \mathrm{SrRuO}_{3}(\mathrm{BFO} / \mathrm{SRO})$ bilayer grown on $\mathrm{SrTiO}_{3}(001)$ substrates were selected for the experiments. These two specific systems displaying distinct lattice mismatch $(2.4 \%$ for $\mathrm{BFO} / \mathrm{LSMO}$ and $1.0 \%$ for $\mathrm{BFO} / \mathrm{SRO})$ were chosen in order to show that the edge dislocations found in both BFO layers exhibit identical characteristics. A STEM Moiré pattern image created intentionally by choosing a sampling interval close to the periodicity of the crystal lattice of a BFO/LSMO sample is shown in Fig. 1(a). This particular sample has a nominal layer thickness of 11 unit cells for LSMO and approximately $100 \mathrm{~nm}$ for BFO and exhibits atomically abrupt and highly controlled epitaxial $\mathrm{LSMO} / \mathrm{SrTiO}_{3}$ and $\mathrm{BFO} / \mathrm{LSMO}$ interfaces. Besides, a high density of dislocations is readily visible from the local bend-

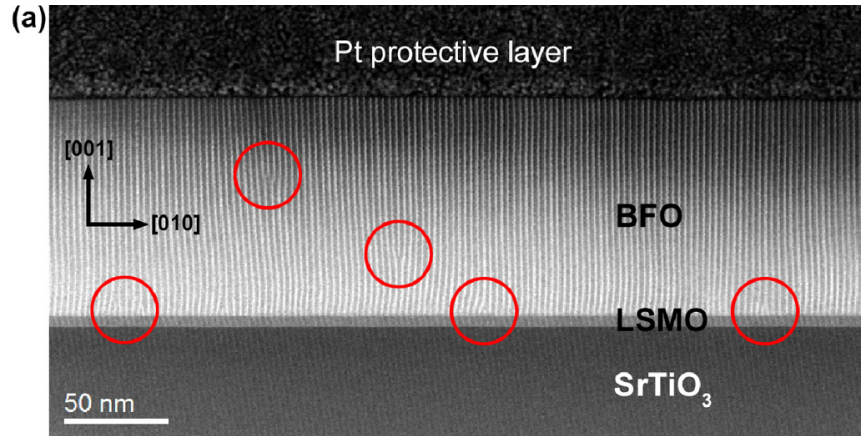

(b)

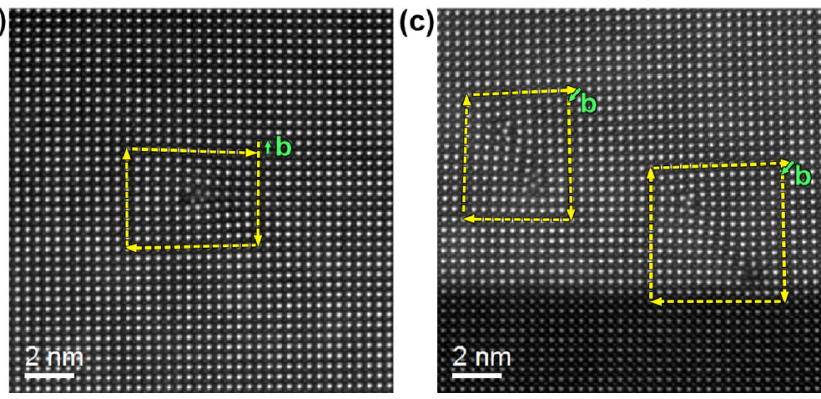

FIG. 1. Representative STEM images of a BFO/LSMO bilayer grown on a $\mathrm{SrTiO}_{3}(001)$ substrate. (a) HAADF-STEM Moiré image of the BFO/LSMO bilayer exhibiting a high density of edge dislocations (highlighted with red circles). (b) A single edge dislocation located in the middle of the BFO layer. The Burgers circuit is shown in yellow and the resulting Burgers vector is indicated with a green arrow. (c) Two pairs of edge dislocations located near the BFO/LSMO interface. Each Burgers circuit encloses a pair of parallel dislocations lying on perpendicular slip planes in close proximity. The resulting Burgers vectors are indicated with green arrows. The crystal orientations shown in (a) apply to all images.

ing of the Moiré fringes both inside the BFO layer and at the $\mathrm{BFO} / \mathrm{LSMO}$ interface [see red circles in Fig. 1(a)]. Figure 1(b) is an atomic-resolution HAADF-STEM image of one single edge dislocation located in the middle of the BFO layer. The Burgers circuit around the dislocation, built following the finish-start right-handed convention [42], is shown in yellow. The Burgers vector obtained by closing the circuit (green arrow) is $\mathbf{b}=[001]$, and the dislocation line $\boldsymbol{\xi}$ runs along the [100] direction. Nevertheless, edge dislocations in BFO commonly appear in pairs. Thus, pairs of parallel edge dislocations laying either on perpendicular or parallel slip planes in close proximity are often observed adjacent to the BFO/LSMO and BFO/SRO interfaces. In Fig. 1(c), two pairs of edge dislocations located near the BFO/LSMO interface are displayed. Each Burgers circuit encloses a pair of parallel dislocations lying on perpendicular slip planes with Burgers vectors $\mathbf{b}=[00-1]$ and [0-10], thus resulting in a global Burgers vector $\mathbf{b}=[0-1-1]$.

Likewise, edge dislocations in the BFO/SRO sample appear in pairs with a global Burgers vector $\mathbf{b}=[0-1-1]$, as shown in the HAADF and annular bright-field (ABF) STEM images of Figs. 2(a) and 2(b), respectively. Here, in order to improve the signal-to-noise ratio, the simultaneous HAADF and $\mathrm{ABF}$ images were obtained as an average of a series consisting of 15 frames, after both rigid and nonrigid registration using the SMART ALIGN software [43]. Due to its 

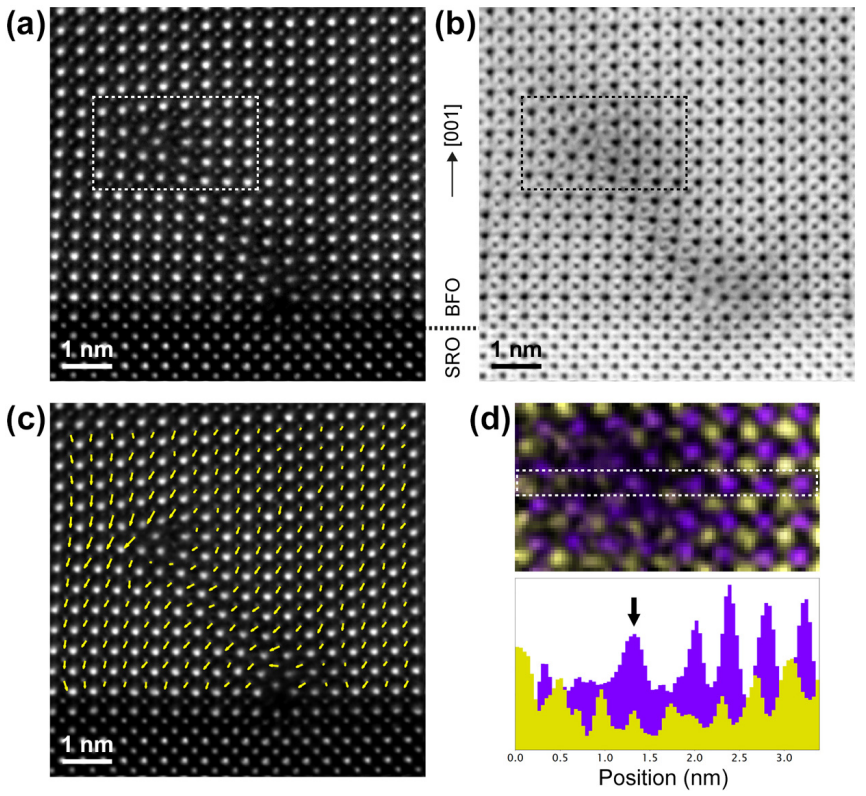

FIG. 2. Representative atomic resolution and chemical mapping images of a $\mathrm{BFO} / \mathrm{SRO}$ bilayer grown on a $\mathrm{SrTiO}_{3}(001)$ substrate. (a) HAADF-STEM image of the BFO/SRO bilayer along the [100] zone axis obtained as an average of a time series consisting of 15 frames. Two parallel edge dislocations lying on perpendicular slip planes are identified nearby the BFO/SRO interface. (b) Corresponding averaged $\mathrm{ABF}$ image of the same area. (c) Polarization map superimposed to the HAADF-STEM image. (d) Elemental maps calculated from the EDX spectrum image acquired in the dashed area specified in the HAADF and ABF images. The (purple) Bi and (yellow) Fe maps were obtained using the $\mathrm{Bi}-\mathrm{M} \alpha 1$ and $\mathrm{Fe}-\mathrm{K} \alpha$ peaks, respectively. Intensity line profiles averaged over seven pixels were extracted from the elemental maps along a line perpendicular to the slip plane (marked by a white dashed box); they uncover the presence of Fe atoms in the dislocation core column.

strong sensitivity to the atomic number, the HAADF averaged image of Fig. 2(a) reveals that the cores of the edge dislocations are likely to be mainly composed of $\mathrm{Bi}$ atoms. Likewise, bright atomic columns were also identified in the majority of the dislocation cores present in the BFO/LSMO sample; see Figs. 1(b) and 1(c). A polarization map is plotted superimposed to the HAADF-STEM image in Fig. 2(c) (see Sec. III A for details). It shows an enhancement of the inplane polarization component between the dislocation cores resulting from the large tensile (compressive) strains in the [010] ([001]) direction in this region, in good agreement with previous reports [32]. In the ABF-STEM image of Fig. 2(b), the strain fields around and between the dislocations are revealed by a darker contrast. ABF-STEM is very sensitive to diffraction contrast and to any effect that leads to a dechanneling of the incident beam. Thus, the strain fields of the dislocations are readily detected by ABF-STEM imaging through the associated displacement of the adjacent atomic sites and the subsequent dechanneling of the electron beam from the atomic columns.

The composition of the edge dislocation cores was assessed by EDX mapping. Figure 2(d) shows the (purple) Bi and (yellow) Fe elemental maps calculated from the EDX spectrum

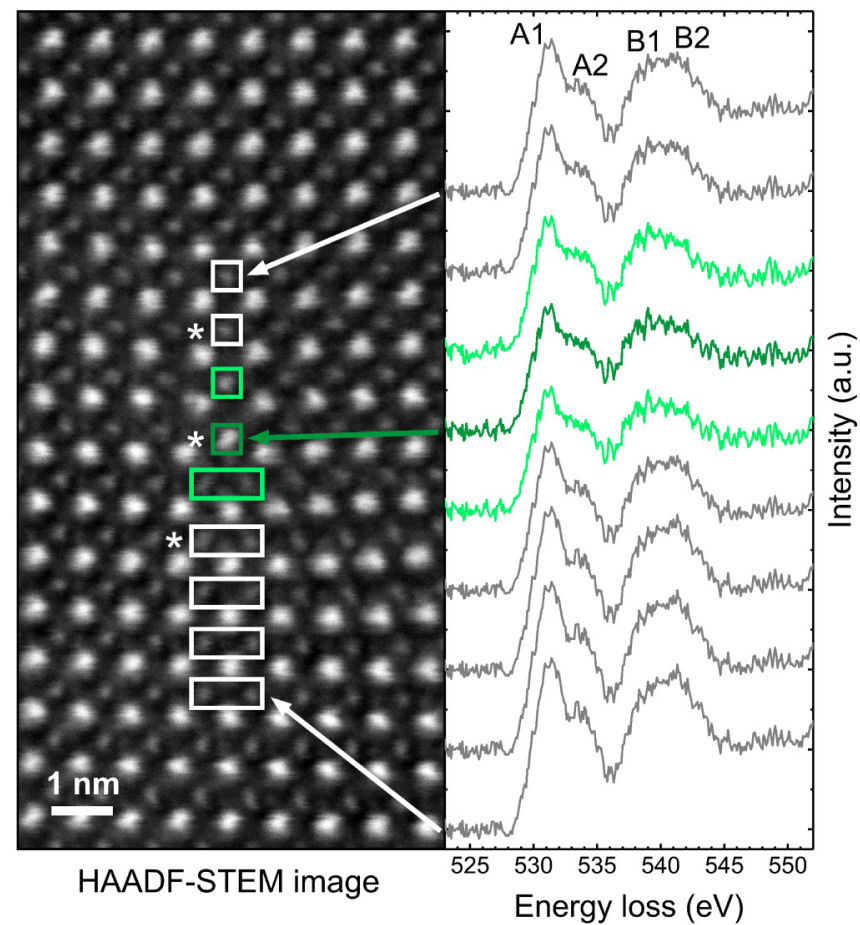

FIG. 3. O- $K$ edge EEL spectra acquired across an edge dislocation core found in the BFO/SRO bilayer sample. The spectra were obtained from the areas indicated by the boxes. The core spectrum is highlighted in dark green, while the spectra immediately above and below the dislocation core are shown in light green. The gray spectra correspond to more distant positions from the dislocation core.

image acquired in the dashed area specified in the HAADF and ABF images. As revealed in the EDX maps of Fig. 2(d), the core of the edge dislocation is mainly composed of $\mathrm{Bi}$ atoms. However, the intensity line profiles (averaged over seven pixels) obtained from the white dashed area reveal the presence of $\mathrm{Fe}$ at the core of the dislocation. Its position is highlighted by a black arrow.

To explore the bonding characteristics of the atoms located at and near the dislocation cores, several EEL spectra were collected and summed over predefined boxes. Thus, Fe- $L_{2,3}$ and $\mathrm{O}-K$ EEL spectra were measured across a line perpendicular to the slip plane, as schematically indicated by the boxes in Fig. 3. While no significant changes were observed in the $L_{2}$ and $L_{3}$ white lines, small spectral changes were detected in the O- $K$ EEL spectra, as seen in Fig. 3 and discussed below. The core spectrum is highlighted in dark green, while the spectra immediately above and below the dislocation core are shown in light green. The spectra collected at more distant positions are shown in gray. All spectra can be divided into two sections: a prepeak region from 528 to $536 \mathrm{eV}$ and a postedge peak from 536 to $545 \mathrm{eV}$. Each region contains two dominant peaks. Thus, at lower energies, the prepeak exhibits a leading peak (labeled A1) at $531 \mathrm{eV}$ and a smaller one at $534 \mathrm{eV}$ (labeled A2). The A1 peak is caused by hybridization of unoccupied O $2 p$ orbitals with Fe $3 d$ orbitals [44-46]. However, the origin of the smaller A2 peak is somewhat ambiguous. Thus, it was suggested that the formation of A2 structures is likely related to interactions between unoccupied 


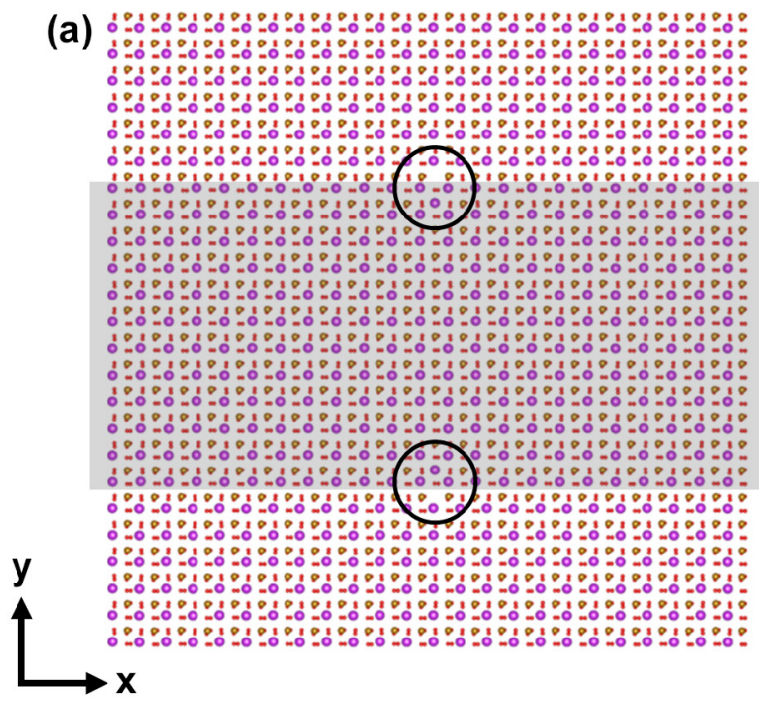

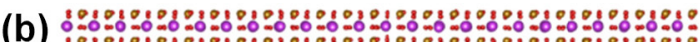

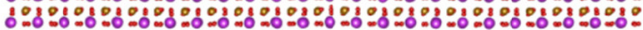

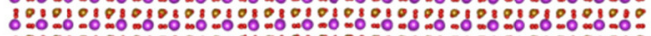

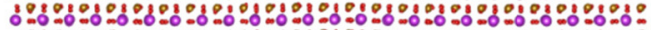

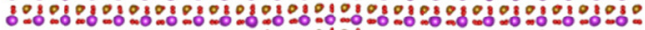

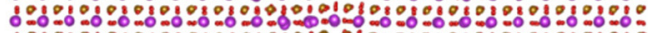

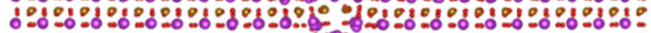

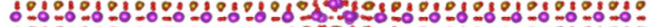

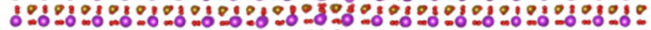

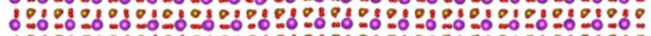

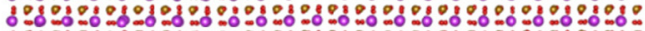

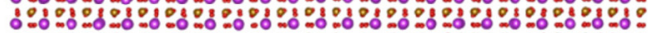

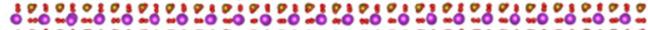

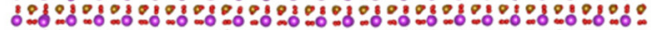

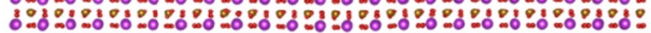
10ำ

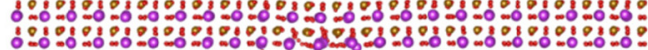

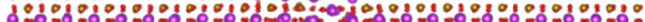

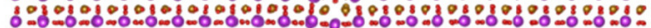

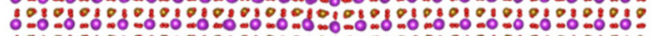

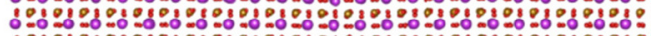

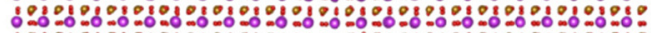

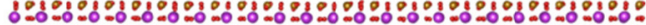

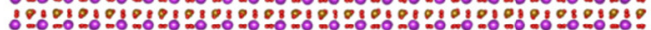

(c)

Model 1

Core: $12 \mathrm{Bi}$ atoms

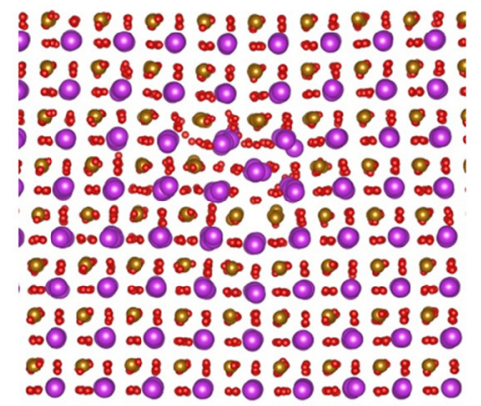

(d)

Core: $8 \mathrm{Bi}$ atoms $+4 \mathrm{Fe}$ atoms

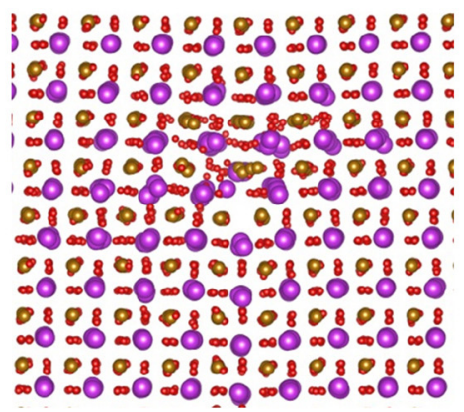

(e) Model 3

Core: $4 \mathrm{Bi}$ atoms $+8 \mathrm{Fe}$ atoms

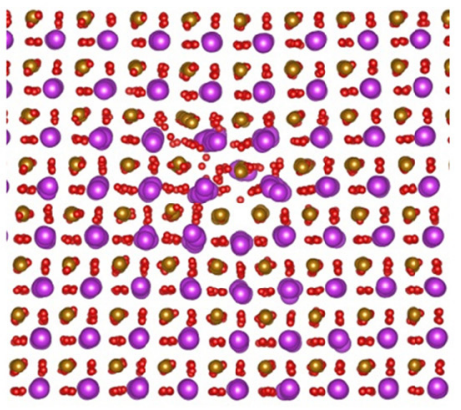

FIG. 4. (a) Initial configuration for the dislocation model used for the molecular dynamics simulation. (b) Optimized configuration of the two dislocation cores obtained using the $\mathrm{BV}$ potential of $\mathrm{BiFeO}_{3}$ [36]. (c)-(e) Optimized configurations of the dislocation core section for the three models obtained after molecular dynamics simulations. The $\mathrm{Bi}, \mathrm{Fe}$, and $\mathrm{O}$ atoms are shown in purple, yellow, and red, respectively.

$\mathrm{O} 2 p$ and $\mathrm{Bi} 5 d$ states [44] or $\mathrm{Bi} 6 s p$ states [47]. Also, Sæterli et al. [45] found that there are mainly Bi states of $p$ character involved in the A2 peak, although some Fe $d$ states were located in this range, too. In the postedge region, two broad peaks can be identified at $539 \mathrm{eV}$ (labeled B1) and 541 $\mathrm{eV}$ (labeled B2) $[44,45,48]$. They stem from transitions to the hybridized $\mathrm{O} 2 p$ and $\mathrm{Fe} 4 s p$ states and are related to the $\mathrm{Fe}$ local coordination set up by the nearest oxygen neighbors and their bonding topology $[49,50]$. Besides, as raised by Sæterli et al. [45], this region might also have some contribution from covalent bonding between $\mathrm{O} 2 p$ and Bi states.

Although the spectral changes through the probing path across the dislocation core are very subtle, the following features can be identified:

(i) The splitting of the A1 and A2 peaks is well defined far from the dislocation core (gray spectra), while it appears blurred (or broadened) in the vicinity of the core (green spectra).

(ii) The B1 peak is higher than the B2 peak at (and near) the dislocation core (green spectra), while this trend is reversed at farther positions (gray spectra).

Note here that the spectral features of the O- $K$ EEL spectra at and near the dislocation core (green spectra) strongly resemble those observed for the highly strained pseudotetragonal BFO phase [48,51].

Subsequently, a three-dimensional (3D) atomic model of the BFO edge dislocation was created in order to assess the effect of nonstoichiometry detected at the dislocation cores on the magnetoelectric properties. Thus, an initial configuration for the model was generated using the experimental lattice parameters of BFO [48] with a $24 \times 24 \times 12$ supercell. Then, a slab of atoms with dimensions $1 \times 11.5 \times 12$ was deleted from the middle part of the supercell and the remaining 23 layers [gray area in Fig. 4(a)] were redistributed uniformly along $x$ to fill the void created due to the deletion of the aforementioned atoms. Additionally, at the two core positions at each side of the stretched gray portion [circled areas in Fig. 4(a)], the Fe-O atomic columns were substituted by $\mathrm{Bi}$ atomic columns and the neighboring oxygen atomic columns were deleted, as described in Lubk et al. [32]. Thus, the atomic configuration in Fig. 4(a) was obtained. Afterwards, this configuration was used as input for the molecular dynamics simulations with BV model potential. This model potential is parametrized from first-principles calculations and is able to reproduce the temperature-driven phase transitions of bulk BFO and the energies of various domain walls [36]. The final 
(a)
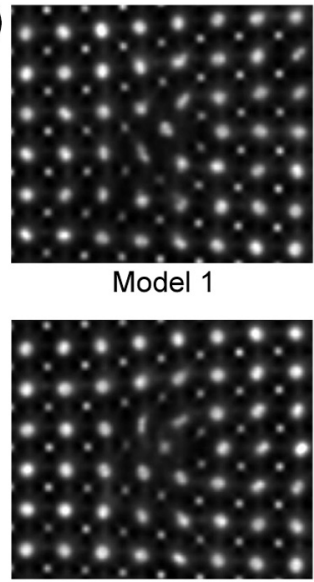

Model 2

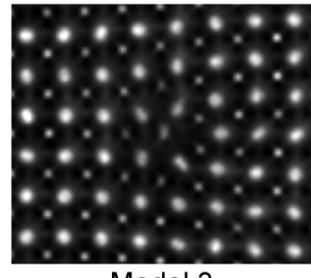

Model 3 (b)

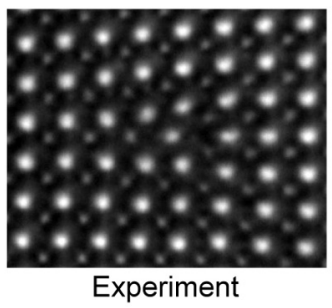

(c)

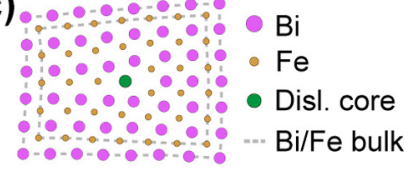

(d)

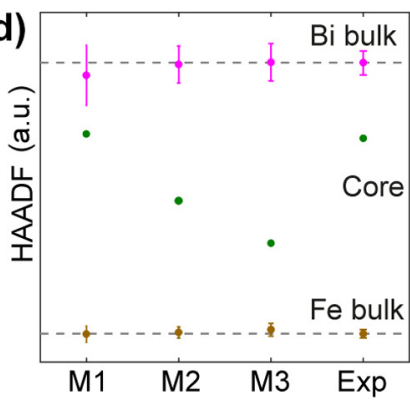

FIG. 5. Comparison between simulated HAADF-STEM images for (a) the three different structural models and (b) the experimental data. (c) Scheme of the edge dislocation with dashed lines indicating the (pink) Bi and (yellow) $\mathrm{Fe}$ atoms used to obtain the bulk-averaged HAADF intensities. (d) Averaged HAADF intensities obtained from the simulated images of each model (M1, M2, and M3) and the experimental data, calculated following the scheme given in (c). The $\mathrm{Bi}$ bulk is represented in pink, Fe bulk in yellow, and core in green.

atomic configuration obtained after dynamic optimization is shown in Fig. 4(b) and verifies the applicability of the BV potential on simulating edge dislocation structures, given the similarity of the optimized structures with the experimental images; see, e.g., Fig. 2(a).

Three atomistic models of edge dislocations accommodating different $\mathrm{Bi} / \mathrm{Fe}$ concentrations were derived following the previously described molecular dynamics steps. In particular, the bottom dislocation core of Fig. 4(a) was selected because of its resemblance-with regard to the crystal orientation-to the experimental results. Then, the composition of the core atomic column was altered in order to accommodate the three following $\mathrm{Bi} / \mathrm{Fe}$ atomic ratios and the models were optimized by molecular dynamics:

Model 1: dislocation core containing $12 \mathrm{Bi}$ atoms;

Model 2: dislocation core containing $8 \mathrm{Bi}$ atoms and $4 \mathrm{Fe}$ atoms; and

Model 3: dislocation core containing $4 \mathrm{Bi}$ atoms and $8 \mathrm{Fe}$ atoms.

The optimized atomistic models are shown in Figs. 4(c)4(e). They all show remarkably similar atomic displacements around the dislocation core.

HAADF-STEM simulations were carried out for each model in order to benchmark them against the experimental data. Figure 5 shows the simulated HAADF-STEM images of the models as well as an experimental image of an edge

dislocation. The latter is indeed an extract of the averaged HAADF-STEM image of Fig. 2(a). In order to estimate the thickness of the sample in Fig. 5(b), the intensity ratio $I_{\mathrm{Bi}} / I_{\mathrm{Fe}}$ obtained in regions far from the dislocation core [highlighted by gray dashed lines in the scheme of Fig. 5(c)] was compared to the $I_{\mathrm{Bi}} / I_{\mathrm{Fe}}$ values of a thickness series performed on bulk BFO. The resulting estimated thickness of $33 \mathrm{~nm}$ was thereafter used to simulate the HAADF-STEM images of all three dislocation models. The graph in Fig. 5(d) compares the averaged HAADF intensities extracted from the simulated images and the experimental data. Specifically, the Bi-bulk and Fe-bulk values were obtained by averaging the intensities of the $\mathrm{Bi}$ and $\mathrm{Fe}$ columns along the dashed gray lines shown in Fig. 5(c). The error bars are the standard deviations calculated by comparing the individual atom columns along the gray dashed lines with the averaged value. As expected, the HAADF intensity of the core atomic column decreases with decreasing $\mathrm{Bi} / \mathrm{Fe}$ ratio, i.e., from Model 1 to Model 3 (green data points). However, one would expect that the core of the model containing only Bi atoms (M1) should exhibit an intensity value comparable to that of the Bi bulk. This is not the case as the displacement of the atoms at the dislocation core leads to a dechanneling of the electron beam from the misaligned cation columns and consequently to an intensity decrease. Therefore, a quantitative analysis based on comparing experimental and simulated HAADF-STEM images of dislocation cores can be misleading.

We compared our EELS experimental spectra with theoretical energy-loss near-edge structures (ELNES) to get a better insight into the effect of Bi substitution by $\mathrm{Fe}$ on the $\mathrm{O}-K$ edge fine structure. For this purpose, three spectra at three different positions were calculated for each structural model. These positions are indicated with asterisks in Fig. 3 and correspond to the atomic columns at the dislocation core and two unit cells above and below the core. For easier comparison with the calculated spectra, the experimental data were fitted with multiple Gaussians. Figure 6 shows the experimental data, the corresponding cumulative Gaussian fit, and the calculated spectra for all three models. The largest difference between the three models can be seen in the postedge region of the (green) spectra obtained at the dislocation cores. Thus, the postedge peak of Models 1 and 2 is peaked at lower energies and is distinctly less intense than that of Model 3, which agrees better with the experimental spectrum. A better agreement regarding the $\mathrm{B} 1 \mathrm{~B} 2$ intensity ratio is also found between the experimental data and Model 3. Far from the dislocation core, the (gray) spectra corresponding to the experimental data and Model 3 exhibit an intensity ratio $\mathrm{B} 1: \mathrm{B} 2<1$. On the contrary, for the equivalent (gray) spectra of Model 1 (top) and Model 2 (bottom), the B1 peak appears more intense relative to the B2 peak, in clear disagreement with the experimental data. Hence, the EELS calculations further support the presence of $\mathrm{Fe}$ in the BFO dislocation cores.

The analysis of the projected density of (unoccupied) states (PDOS) allows rationalizing the behavior of the EEL spectra of Model 3. The PDOS calculated for Model 3 at three different atomic positions, namely at the core region and two unit cells below and above the core, are shown in Fig. 7. It shows that in all cases, the leading prepeak A1 (region from 1 to $3 \mathrm{eV}$ ) is originated by hybridization of $\mathrm{O} 2 p$ and $\mathrm{Fe}$ 


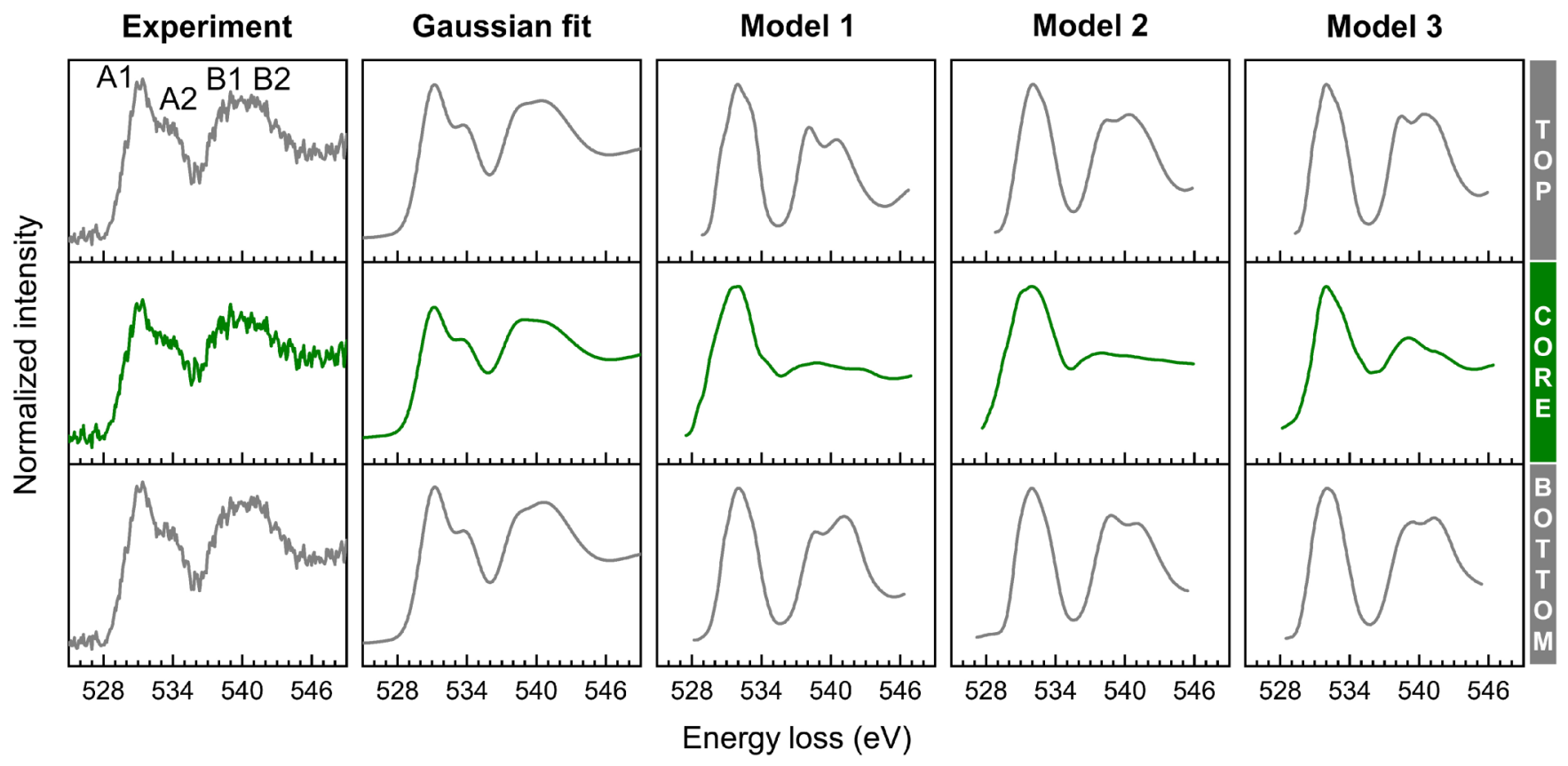

FIG. 6. Comparison of the O- $K$ edge EEL spectra obtained from the three different dislocation core models with dissimilar Bi/Fe atomic ratios together with the experimentally acquired $\mathrm{O}-K$ edge spectra. For easier comparison with the calculated spectra, the experimental data were fitted with multiple Gaussians.

$3 d$ states. In the region from 3 to $7 \mathrm{eV}$, there is contribution from $\mathrm{Bi} 6 p$ and $\mathrm{O} 2 p$ states resulting in the A2 peak, in good agreement with Sæterli et al. [45]. There is, however, an important difference between the PDOS of the core and top/bottom positions. While the $\mathrm{Bi} 6 p$ peak around $6 \mathrm{eV}$ is very sharp for the bottom and top positions [Fig. 7(b)], it is redistributed and broadened at the dislocation core (containing a mixture of $\mathrm{Bi}$ and $\mathrm{Fe}$ atoms along the column) due to an enhancement in the bonding between the oxygen and $\mathrm{Bi}$ atoms. This results in the blurred (or broadening of the) A2 peak observed at the core position.

In the postedge region, the two peaks B1 and B2 can be attributed to $\mathrm{O} 2 p$ states hybridized with $\mathrm{Fe} 4 s p$ and covalently bonded to Bi states (see Supplemental Material [52]). Additionally, the $\mathrm{Fe} 4 s$ states around $11 \mathrm{eV}$ for the bottom/top positions [Fig. 7(e)] are again redistributed at a lower energy $(8 \mathrm{eV})$ in the core. This also explains why the postedge region is broadened and less peaked at the dislocation core.

In analogy to the system investigated by Sugiyama et al. [40], a ferromagnetic behavior is expected for the dislocations in $\mathrm{BiFeO}_{3}$ due to uncompensated spins of the $\mathrm{Fe}$ cations along the dislocation cores. Since in the TEM cross-sectional specimens most of the dislocation cores appear parallel to the optical axis of the electron microscope, we tilted the sample by $15.6^{\circ}$ in order to investigate the magnetic properties of the dislocation cores. In this way, a component of the dislocation core magnetization is projected into the plane normal to the observation direction and can be detected by off-axis electron holography as a phase shift of the electron wave function. In Fig. 8(a), an electron hologram of the $\mathrm{BFO} / \mathrm{LSMO}$ bilayer grown on a $\mathrm{SrTiO}_{3}(001)$ substrate is shown. A scheme of the experimental geometry is shown in the inset. The reconstructed mean inner potential (MIP) and the magnetic phase shift (see Sec. III A for details) for the area marked by the white dashed box in Fig. 8(a) are shown in Figs. 8(b) and 8(c), respectively. Interestingly, while no clear features are seen in the MIP map, the magnetic phase shift map displays a dipolarlike feature in the BFO layer, as evidenced from the line profile of the magnetic phase shift [see Fig. 8(e)] obtained along the blue rectangular box in Fig. 8(c). In particular, the shape of the profile can be interpreted as the result of the superimposition of two magnetic dipoles centered in the positions marked by the red lines, thus separated by about $15 \mathrm{~nm}$. In Fig. 8(f), a magnified view of the magnetic phase shift of the two dislocation cores is given together with the phase contours of the amplified (24x) phase, which is compatible with two magnetic dipoles oriented in antiparallel configuration. A high-resolution HAADF-STEM of the same portion of the sample is shown in Fig. 8(d) overlaid to the rotation map obtained by geometrical phase analysis (GPA). The two dislocation cores generating the signal in the magnetic phase shift can be immediately identified as singularity points in the color-coded map. Thus, it appears that the dislocation cores locally break the system symmetry and the uncompensated $\mathrm{Fe}$ spins along the dislocation core give rise to a magnetic signal, in analogy with recent findings of ferromagnetic dislocation cores in otherwise antiferromagnetic $\mathrm{NiO}$ [40]. It is worth mentioning here that in the BFO system, the coupled dislocations appearing at distances below $15 \mathrm{~nm}$ and displaying ferromagnetic behavior are in an antiferromagnetic configuration. Further, our electron holography data do not reveal any magnetic phase shift from the 4.3-nm-thick LSMO buffer layer. This is in good agreement with the work of Borges et al. [53], which reported that in thin LSMO films (i.e., $\leqslant 4 \mathrm{~nm}$ ), the magnetization falls rapidly from 0 to $285 \mathrm{~K}$ and no ferromagnetic signal is detected at room temperature. This behavior was attributed to the formation of magnetic 

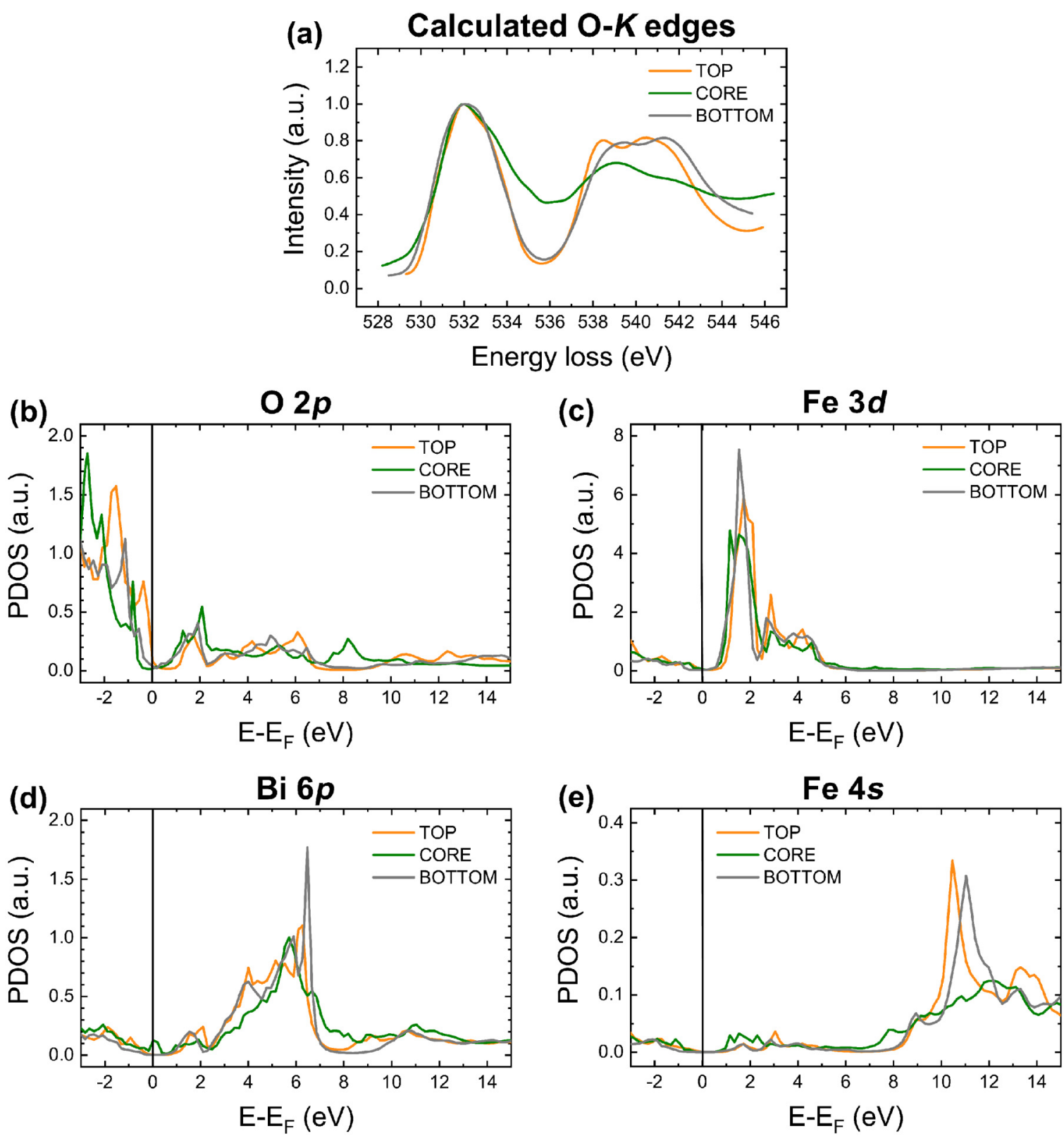

FIG. 7. Energy-loss near-edge structures and projected density of states (PDOS) calculated for Model 3 at the dislocation core and "bottom" and "top" positions (two unit cells below and above the core, as defined in the text). Contributions from the different atomic orbitals for the O, $\mathrm{Fe}$, and $\mathrm{Bi}$ atomic species are given in separate panels.

dead layers at the different interfaces resulting from weakly coupled noncollinear spins.

\section{METHODS}

\section{A. Experimental methods}

Thin $\mathrm{BiFeO}_{3}$ films with thicknesses of 50 to $100 \mathrm{~nm}$ were grown on either $\mathrm{SrRuO}_{3}(\mathrm{SRO})$ or $\mathrm{La}_{0.7} \mathrm{Sr}_{0.3} \mathrm{MnO}_{3}$ (LSMO) buffer layers on top of $\mathrm{SrTiO}_{3}(001)$ single-crystal substrates by pulsed laser deposition while utilizing high-pressure reflection high-energy electron diffraction (RHEED) to verify the growth mechanism. All SRO, LSMO, and BFO layers were grown epitaxially on the $\mathrm{SrTiO}_{3}$ substrate at $670-690^{\circ} \mathrm{C}$ in 100-150 mTorr of oxygen pressure. The laser energy was fixed at $1.5 \mathrm{~mJ} / \mathrm{cm}^{2}$ for all materials. After the growth, the samples were cooled to room temperature in 760 Torr oxygen ambient at a rate of $5{ }^{\circ} \mathrm{C} / \mathrm{min}$. A postannealing process was carried out in oxygen ambient at $400{ }^{\circ} \mathrm{C}$ for $1 \mathrm{~h}$ to ensure that the samples were fully oxidized. More details about the film growth conditions can be found elsewhere $[32,54]$.

Electron transparent samples for transmission electron microscopy were prepared in cross-section geometry by means of a FEI Helios NanoLab 600i and a FEI Helios NanoLab $450 \mathrm{~S}$ focused ion beam (FIB) operated at accelerating voltages of 30 and $5 \mathrm{kV}$. The samples were cut parallel to the (100) planes of the $\mathrm{SrTiO}_{3}$ substrate.

High-angle annular dark-field (HAADF) and simultaneous annular bright-field (ABF) scanning transmission electron microscopy (STEM), energy dispersive x-ray (EDX) 
(a)

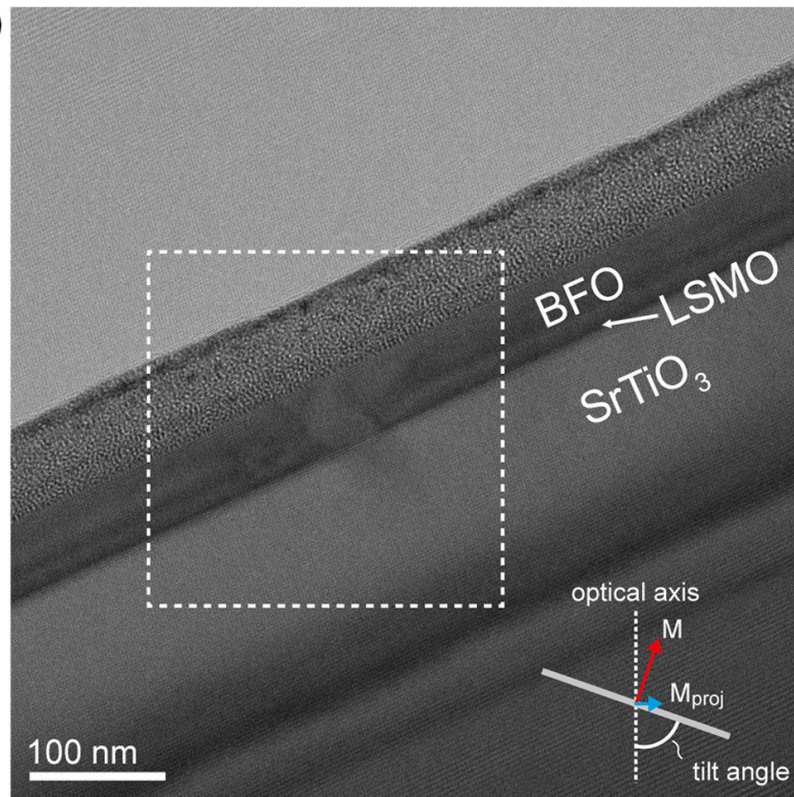

(b)

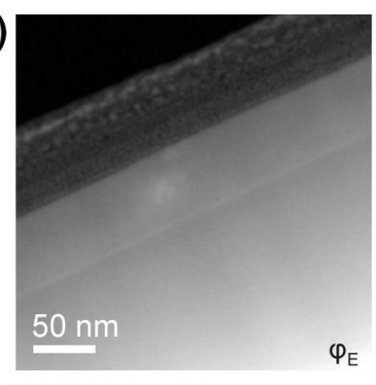

(d)

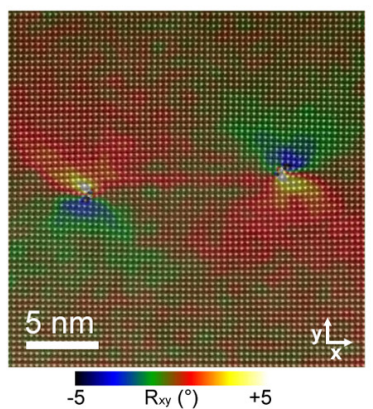

(c)

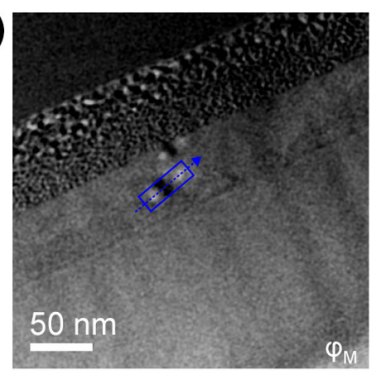

(e)

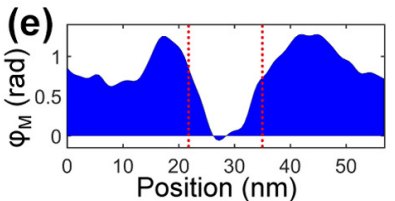

(f)

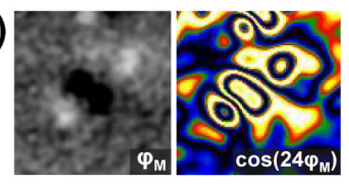

FIG. 8. Off-axis electron holography investigation of a BFO/LSMO bilayer grown on a $\mathrm{SrTiO}_{3}(001)$ substrate acquired with a tilt of $15.6^{\circ}$. (a) Electron hologram acquired at $370 \mathrm{~V}$. (b) Mean inner potential and (c) magnetic contribution to the electron phase shift. (d) Rotation map superimposed to the atomic-resolution HAADF-STEM image showing the two dislocation cores at a distance of about $15 \mathrm{~nm}$. This image was rotated with respect to the other panels in order to align the atomic columns with the edges of the image. (e) Profile of the magnetic phase shift along the direction connecting the two dislocation cores [marked in blue in (c)]. The positions of the cores are marked by the red lines. (f) Magnified view of the magnetic phase shift of the two dislocation cores and phase contours of the amplified $(24 \mathrm{x})$ phase. The two dislocation cores display a dipolarlike behavior.

spectroscopy, and electron energy-loss spectroscopy (EELS) measurements were performed using a double spherical aberration-corrected JEOL JEM-ARM200F microscope operated at $200 \mathrm{kV}$, and equipped with a JEOL Dry SD100GV

silicon drift detector with $100 \mathrm{~mm}^{2}$ detection area for EDX analysis, and a Gatan Enfinium EELS spectrometer. HAADF and ABF-STEM simultaneous imaging were carried out setting a probe semiconvergence angle of $18 \mathrm{mrad}$ and collecting angles of 90-170 and 9-18 mrad, respectively. In typical operating conditions for the EDX and EELS experiments described in this work, the electron probe current was kept below $200 \mathrm{pA}$ to avoid radiation damage. The elemental maps shown in Fig. 2(c) were calculated from the EDX spectrum image using the $\mathrm{Bi}-\mathrm{M} \alpha 1$ and $\mathrm{Fe}-\mathrm{K} \alpha$ peaks located at 2.423 and $6.400 \mathrm{keV}$, respectively. The dimensions of the EDX spectrum image are $87 \times 50$ pixels, with $0.04 \mathrm{~nm}$ pixel spacing and a dwell of $0.1 \mathrm{~s}$ per pixel. For the EELS data acquisition, the convergence and collection semiangles were set to 25.3 and $33 \mathrm{mrad}$, respectively. A dispersion of $0.1 \mathrm{eV}$ per channel was selected. All spectra were background subtracted by fitting a decaying power-law function to an energy window just in front of the $\mathrm{O}$ core-loss edge onset. Multivariate statistical analysis based on principal component analysis (PCA) was used to remove noise from the low-count experimental EELS data [55].

The off-axis electron holography data were acquired in Lorentz mode using a FEI Titan Themis microscope operated at $300 \mathrm{kV}$ and equipped with a FEI Ceta2 16 Mpixel CMOS camera. The holograms were acquired setting the exposition time to $10 \mathrm{~s}$ and applying to the electrostatic biprism a potential of $370 \mathrm{~V}$, thus giving a spatial resolution of $3.3 \mathrm{~nm}$ and a fringe contrast of $22 \%$. In order to separate the electrostatic contribution to the phase shift (or mean inner potential, MIP) from the magnetic phase shift, we applied the separation method [56], i.e., we acquired two holograms at reversed incident electron beam directions by manually flipping the sample upside down. The MIP and the magnetic phase shift are then obtained as half of the sum and of the difference of the two phase images, respectively.

Polarization mapping was performed using customdeveloped MATLAB codes. The atomic column positions were fitted over the entire field of view of the time-averaged HAADF-STEM signal, and the displacement vectors were calculated by measuring the off-centering between the Fe-site position and the center of mass of the Bi cell. Thus, the polarization vectors are plotted opposite to the polar displacement of the Fe cations, following the method proposed by Nelson et al. [57]. Geometrical phase analysis (GPA) of the edge dislocations in the high-resolution HAADF-STEM image of Fig. 8(d) was performed by using the GPA software package for DIGITAL MICROGRAPH [58].

\section{B. Computational methods}

The initial configuration for the dislocation model was generated using the experimental lattice parameters of BFO [48] with a $24 \times 24 \times 12$ supercell (see Supplemental Material [52]). The bond-valence atomistic potential [36] designed for BFO was used to perform molecular dynamics simulations. The potentials were implemented in the classical molecular dynamics code LAMMPS [59]. The initial configuration of the dislocation core structure was modified in order to accommodate different $\mathrm{Bi} / \mathrm{Fe}$ concentrations. In particular, three distinct core models were optimized with molecular 
dynamics as described in the main text. The molecular dynamics structural optimization was performed following three steps: (a) gradually decreasing the temperature from 10 to $0 \mathrm{~K}$ by applying a viscous force to each atom; (b) then, gradually increasing the temperature to $300 \mathrm{~K}$ in the NVT ensemble; and (c) equilibrating the system at $300 \mathrm{~K}$. The final optimized configuration was obtained by taking the average of several structures at $300 \mathrm{~K}$.

HAADF-STEM simulations of the three models were performed using the STEM_CELL software, based on the Kirkland code for TEM image simulations [60]. For all the considered models, the simulations were performed using the multislice algorithm by computing the average of 10 frozen phonon configurations, using constant Debye-Waller factors for all the atomic species. In order to estimate the thickness of the sample, a thickness series (thickness range 0$46 \mathrm{~nm}$ ) was performed on bulk BFO with the aim of comparing the intensity ratio $I_{\mathrm{Bi}} / I_{\mathrm{Fe}}$ to the experimental data obtained in regions far from the dislocations, where the dislocation strain fields are negligible. The estimated thickness was 33 $\mathrm{nm}$.

The real-space multiple-scattering code FEFF 9.0 [61] was used for the calculation of the energy-loss near-edge structures (ELNES) of the O- $K$ edge as well as the PDOS at different points above and below the dislocation core along a direction parallel to the extra half plane, i.e., similarly to the experimental data of Fig. 3(a). Each point reflects the integral of all possible oxygen environments around the $\mathrm{Fe}$ atom with a lateral extension of one pseudocubic perovskite unit cell. The scattering potentials were calculated self-consistently over a radius of 5.1-5.3 $\AA$ using HedinLundqvist (local density approximation) self-energies [62]. For the full multiple-scattering (FMS) calculations, the calculations converged for clusters as small as 260 atoms. The spectra and density-of-state calculations presented here were all modeled for 330 atoms in the FMS cluster. The O- $K$ edge spectra were calculated for each oxygen atom surrounding a $\mathrm{Fe}$ atom separately and averaged over a volume consisting of $1 \times 1 \times 12$ pseudocubic perovskite unit cells around the $\mathrm{Fe}$ atomic columns. Additional self-consistent iterations are needed to converge the total and projected density of states from FEFF calculations and we followed the procedure described in Ref. [41] which also includes an additional shift of about $2 \mathrm{eV}$ in the Fermi energy to match the experimental evidence of an electronic band gap in the BFO material.

\section{CONCLUSIONS}

In the present work, we have exhaustively investigated the structural and magnetoelectric properties of edge dislocations found in epitaxial BFO thin films deposited on STO substrates via two different buffer layers, SRO and LSMO. By using a combined atomic-level experimental and theoretical approach, chemical and electronic information of the individual atomic columns located at and near the dislocation cores was obtained. Our results reveal that-in addition to $\mathrm{Bi}-$ small amounts of $\mathrm{Fe}$ atoms are present at $\mathrm{BiFeO}_{3}$ dislocation cores which result in uncompensated $\mathrm{Fe}$ spins along the dislocation line and give rise to a magnetic signal. Thus, our findings suggest the possibility of exploiting these particular defects in antiferromagnetic thin films to achieve ferromagnetic properties beyond those of the corresponding perfect structure.

\section{ACKNOWLEDGMENTS}

The authors are thankful to $\mathrm{Pu} \mathrm{Yu}$ and Ramamoorthy Ramesh for the samples used in this study. This research was supported by the Swiss National Science Foundation (SNSF) with Project No. 200021_147105. M.C. and M.D.R. thank the NCCR-MARVEL project funded by the Swiss National Science Foundation (SNSF) for the financial support. A.M.R was supported by the Department of Energy under Grant No. DE-FG02-07ER46431. Access to the TEM facility at the IBM Research-Zürich (Switzerland) under the IBM/Empa Master Joint Development Agreement is gratefully acknowledged.
[1] W. Eerenstein, N. D. Mathur, and J. F. Scott, Multiferroic and magnetoelectric materials, Nature (London) 442, 759 (2006).

[2] K. F. Wang, J.-M. Liu, and Z. F. Ren, Multiferroicity: The coupling between magnetic and polarization orders, Adv. Phys. 58, 321 (2009).

[3] N. A. Spaldin and M. Fiebig, The renaissance of magnetoelectric multiferroics, Science 309, 391 (2005).

[4] J. F. Scott, Ferroelectric Memories (Springer-Verlag, Berlin, 2000).

[5] G. A. Smolenskii and I. E. Chupis, Ferroelectromagnets, Sov. Phys. Usp. 25, 475 (1982).

[6] C. Michel, J.-M. Moreau, G. D. Achenbach, R. Gerson, and W. J. James, The atomic structure of $\mathrm{BiFeO}_{3}$, Solid State Commun. 7, 701 (1969).

[7] F. Kuebel and H. Schmid, Structure of a ferroelectric and ferroelastic monodomain crystal of the perovskite $\mathrm{BiFeO}_{3}$, Acta Cryst. B 46, 698 (1990).

[8] D. Lebeugle, D. Colson, A. Forget, and M. Viret, Very large spontaneous electric polarization in $\mathrm{BiFeO}_{3}$ single crystals at room temperature and its evolution under cycling fields, Appl. Phys. Lett. 91, 022907 (2007).

[9] V. V. Shvartsman, W. Kleemann, R. Haumont, and J. Kreisel, Large bulk polarization and regular domain structure in ceramic $\mathrm{BiFeO}_{3}$, Appl. Phys. Lett. 90, 172115 (2007).

[10] S. V. Kiselev, R. P. Ozerov, and G. S. Zhdanov, Detection of magnetic order in ferroelectric $\mathrm{BiFeO}_{3}$ by neutron diffraction, Sov. Phys. Dokl. 7, 742 (1963).

[11] I. E. Dzialoshinskii, Thermodynamic theory of weak ferromagnetism in antiferromagnetic substances, Sov. Phys. JETP 5, 1259 (1957).

[12] T. Moriya, Anisotropic superexchange interaction and weak ferromagnetism, Phys. Rev. 120, 91 (1960).

[13] C. Ederer and N. A. Spaldin, Weak ferromagnetism and magnetoelectric coupling in bismuth ferrite, Phys. Rev. B 71, 060401(R) (2005).

[14] R. Ramesh and N. A. Spandin, Multiferroics: Progress and prospects in thin films, Nat. Mater. 6, 21 (2007). 
[15] J. Wang, J. B. Neaton, H. Zheng, V. Nagarajan, S. B. Ogale, B. Liu, D. Viehland, V. Vaithyanathan, D. G. Schlom, U. V. Waghmare, N. A. Spaldin, K. M. Rabe, M. Wuttig, and R. Ramesh, Epitaxial $\mathrm{BiFeO}_{3}$ multiferroic thin film heterostructures, Science 299, 1719 (2003).

[16] M. Trassin, J. D. Clarkson, S. R. Bowden, J. Liu, J. T. Heron, R. J. Paull, E. Arenholz, D. T. Pierce, and J. Unguris, Interfacial coupling in multiferroic/ferromagnet heterostructures, Phys. Rev. B 87, 134426 (2013).

[17] J. T. Heron, M. Trassin, K. Ashraf, M. Gajek, Q. He, S.Y. Yang, D. E. Nikonov, Y-H. Chu, S. Salahuddin, and R. Ramesh, Electric-Field-Induced Magnetization Reversal in a Ferromagnet-Multiferroic Heterostructure, Phys. Rev. Lett. 107, 217202 (2011)

[18] I. Sosnowska, T. Peterlin-Neumaier, and E. Steichele, Spiral magnetic ordering in bismuth ferrite, J. Phys. C: Solid State Phys. 15, 4835 (1982).

[19] I. Gross, W. Akhtar, V. Garcia, L. J. Martínez, S. Chouaieb, K. Garcia, C. Carrétéro, A. Barthélémy, P. Appel, P. Maletinsky, J.-V. Kim, J. Y. Chauleau, N. Jaouen, M. Viret, M. Bibes, S. Fusil, and V. Jacques, Real-space imaging of non-collinear antiferromagnetic order with a single-spin magnetometer, Nature (London) 549, 252 (2017).

[20] I. Sosnowska, W. Schäfer, W. Kockelmann, K. H. Andersen, and I. O. Troyanchuk, Crystal structure and spiral magnetic ordering of $\mathrm{BiFeO}_{3}$ doped with manganese, Appl. Phys. A 74, S1040 (2002).

[21] V. R. Palkar, D. C. Kundaliya, S. K. Malik, and S. Bhattacharya, Magnetoelectricity at room temperature in the $\mathrm{Bi}_{0.9-x} \mathrm{~Tb}_{x} \mathrm{La}_{0.1} \mathrm{FeO}_{3}$ system, Phys. Rev. B 69, 212102 (2004).

[22] S.-T. Zhang, Y. Zhang, M.-H. Lu, C.-L. Du, Y.-F. Chen, Z.-G. Liu, Y.-Y. Zhu, and N.-B. Ming, Substitution-induced phase transition and enhanced multiferroic properties of $\mathrm{Bi}_{1-x} \mathrm{La}_{x} \mathrm{FeO}_{3}$ ceramics, Appl. Phys. Lett. 88, 162901 (2006).

[23] G. Le Bras, D. Colson, A. Forget, N. Genand-Riondet, R. Tourbot, and $\mathrm{P}$. Bonville, Magnetization and magnetoelectric effect in $\mathrm{Bi}_{1-x} \mathrm{La}_{x} \mathrm{FeO}_{3}(0 \leqslant x \leqslant 0.15)$, Phys. Rev. B 80, 134417 (2009).

[24] M. Campanini, R. Erni, C.-H. Yang, R. Ramesh, and M. D. Rossell, Periodic giant polarization gradients in doped $\mathrm{BiFeO}_{3}$ thin films, Nano Lett. 18, 717 (2018).

[25] X. Yuan, L. Shi, J. Zhao, S. Zhou, J. Guo, S. Pan, X. Miao, and $\mathrm{L}$. Wu, Tunning ferroelectric, dielectric, and magnetic properties of $\mathrm{BiFeO}_{3}$ veramics by $\mathrm{Ca}$ and $\mathrm{Pb}$ co-doping, Phys. Status Solidi B 256, 1800499 (2019).

[26] R. J. Zeches, M. D. Rossell, J. X. Zhang, A. J. Hatt, Q. He, C.-H. Yang, A. Kumar, C. H. Wang, A. Melville, C. Adamo, G. Sheng, Y.-H. Chu, J. F. Ihlefeld, R. Erni, C. Ederer, V. Gopalan, L. Q. Chen, D. G. Schlom, N. A. Spaldin, L. W. Martin, and R. Ramesh, A strain-driven morphotropic phase boundary in $\mathrm{BiFeO}_{3}$, Science 326, 977 (2009).

[27] H. Béa, B. Dupé, S. Fusil, R. Mattana, E. Jacquet, B. WarotFonrose, F. Wilhelm, A. Rogalev, S. Petit, V. Cros, A. Anane, F. Petroff, K. Bouzehouane, G. Geneste, B. Dkhil, S. Lisenkov, I. Ponomareva, L. Bellaiche, M. Bibes, and A. Barthélémy, Evidence for Room-Temperature Multiferroicity in a Compound with a Giant Axial Ratio, Phys. Rev. Lett. 102, 217603 (2009).

[28] D. Mazumdar, V. Shelke, M. Iliev, S. Jesse, A. Kumar, S. V. Kalinin, A. P. Baddorf, and A. Gupta, Nanoscale switching characteristics of nearly tetragonal $\mathrm{BiFeO}_{3}$ thin films, Nano Lett. 10, 2555 (2010).

[29] J. X. Zhang, Q. He, M. Trassin, W. Luo, D. Yi, M. D. Rossell, P. Yu, L. You, C. H. Wang, C.Y. Kuo, J. T. Heron, Z. Hu, R. J. Zeches, H. J. Lin, A. Tanaka, C. T. Chen, L. H. Tjeng, Y.-H. Chu, and R. Ramesh, Microscopic Origin of the Giant Ferroelectric Polarization in Tetragonal-like $\mathrm{BiFeO}_{3}$, Phys. Rev. Lett. 107, 147602 (2011).

[30] Y.-H. Chu, Q. He, C.-H. Yang, P. Yu, L. W. Martin, P. Shafer, and R. Ramesh, Nanoscale control of domain architectures in $\mathrm{BiFeO}_{3}$ thin films, Nano Lett. 9, 1726 (2009).

[31] A. Lubk, M. D. Rossell, J. Seidel, Q. He, S. Y. Yang, Y. H. Chu, R. Ramesh, M. J. Hÿtch, and E. Snoeck, Evidence of Sharp and Diffuse Domain Walls in $\mathrm{BiFeO}_{3}$ by Means of Unit-Cell-Wise Strain and Polarization Maps Obtained with High Resolution Scanning Transmission Electron Microscopy, Phys. Rev. Lett. 109, 047601 (2012).

[32] A. Lubk, M. D. Rossell, J. Seidel, Y. H. Chu, R. Ramesh, M. J. Hÿtch, and E. Snoeck, Electromechanical coupling among edge dislocations, domain walls, and nanodomains in $\mathrm{BiFeO}_{3}$ revealed by unit-cell-wise strain and polarization maps, Nano Lett. 13, 1410 (2013).

[33] J. Přívratská and V. Janovec, Pyromagnetic domain walls connecting antiferromagnetic non-ferroelastic magnetoelectric domains, Ferroelectrics 204, 321 (1997).

[34] J. Př́vratská and V. Janovec, Spontaneous polarization and/or magnetization in non-ferroelastic domain walls: symmetry predictions, Ferroelectrics 222, 23 (1999).

[35] Q. He, C.-H. Yeh, J.-C. Yang, G. Singh-Bhalla, C.-W. Liang, P.-W. Chiu, G. Catalan, L. W. Martin, Y.-H. Chu, J. F. Scott, and R. Ramesh, Magnetotransport at Domain Walls in $\mathrm{BiFeO}_{3}$, Phys. Rev. Lett. 108, 067203 (2012).

[36] S. Liu, I. Grinberg, and A. M. Rappe, Development of a bondvalence based interatomic potential for $\mathrm{BiFeO}_{3}$ for accurate molecular dynamics simulations, J. Phys.: Condens. Matter 25, 102202 (2013).

[37] M. L. Jablonski, S. Liu, C. R. Winkler, A. R. Damoodaran, I. Grinberg, L. W. Martin, A. M. Rappe, and M. L. Taheri, Asymmetric response of ferroelastic domain-wall motion under applied bias, ACS Appl. Mater. Interfaces 8, 2935 (2016).

[38] R. Ranjan, O. Buck, and R. B. Thompson, A study on the effect of dislocation on the magnetic properties of nickel using magnetic NDE methods, J. Appl. Phys. 61, 3196 (1987).

[39] M. J. Sablik, Modeling the effect of grain size and dislocation density on hysteretic magnetic properties in steels, J. Appl. Phys. 89, 5610 (2001).

[40] I. Sugiyama, N. Shibata, Z. Wang, S. Kobayashi, T. Yamamoto, and Y. Ikuhara, Ferromagnetic dislocations in antiferromagnetic NiO, Nat. Nanotechnol. 8, 266 (2013).

[41] M. S. Moreno, K. Jorissen, and J. J. Rehr, Practical aspects of electron energy-loss spectroscopy (EELS) calculations using FEFF8, Micron 38, 1 (2007).

[42] J. P. Hirth and J. Lothe, Theory of Dislocations, second ed. (Wiley, New York, 1982).

[43] L. Jones, H. Yang, T. J. Pennycook, M. S. J. Marshall, S. Van Aert, N. D. Browning, M. R. Castell, and P. D. Nellist, Smart Align - A new tool for robust non-rigid registration of scanning microscopy data, Adv. Struct. Chem. Imaging 1, 8 (2015).

[44] T. J. Park, S. Sambasivan, D. A. Fischer, W.-S. Yoon, J. A. Misewich, and S. S. Wong, Electronic structure and chem- 
istry of iron-based metal oxide nanostructured materials: A NEXAFS investigation of $\mathrm{BiFeO}_{3}, \mathrm{Bi}_{2} \mathrm{Fe}_{4} \mathrm{O}_{9}, \alpha-\mathrm{Fe}_{2} \mathrm{O}_{3}, \gamma-$ $\mathrm{Fe}_{2} \mathrm{O}_{3}$, and $\mathrm{Fe} / \mathrm{Fe}_{3} \mathrm{O}_{4}$, J. Phys. Chem. C 112, 10359 (2008).

[45] R. Sæterli, S. M. Selbach, P. Ravindran, T. Grande, and R. Holmestad, Electronic structure of multiferroic $\mathrm{BiFeO}_{3}$ and related compounds: Electron energy loss spectroscopy and density functional study, Phys. Rev. B 82, 064102 (2010).

[46] K. T. Ko, M. H. Jung, Q. He, J. H. Lee, C. S. Woo, K. Chu, J. Seidel, B. G. Jeon, Y. S. Oh, K. H. Kim, W. I. Liang, H. J. Chen, Y. H. Chu, Y. H. Jeong, R. Ramesh, J. H. Park, and C. H. Yang, Concurrent transition of ferroelectric and magnetic ordering near room temperature, Nat. Commun. 2, 567 (2011).

[47] T. Higuchi, Y.-S. Liu, P. Yao, P.-A. Glans, and J. Guo, Electronic structure of multiferroic $\mathrm{BiFeO}_{3}$ by resonant soft $\mathrm{x}$-ray emission spectroscopy, Phys. Rev. B 78, 085106 (2008).

[48] M. D. Rossell, R. Erni, M. P. Prange, J.-C. Idrobo, W. Luo, R. J. Zeches, S. T. Pantelides, and R. Ramesh, Atomic Structure of Highly Strained $\mathrm{BiFeO}_{3}$ Thin Films, Phys. Rev. Lett. 108, 047601 (2012).

[49] F. M. F. de Groot, J. Faber, J. J. M. Michiels, M. T. Czyżyk, M. Abbate, and J. C. Fuggle, Oxygen $1 s$ x-ray absorption of tetravalent titanium oxides: A comparison with single-particle calculations, Phys. Rev. B 48, 2074 (1993).

[50] N. D. Browning, H. O. Moltaji, and J. P. Buban, Investigation of three-dimensional grain-boundary structures in oxides through multiple-scattering analysis of spatially resolved electron-energy-loss spectra, Phys. Rev. B 58, 8289 (1998).

[51] Y. H. Kim, A. Bhatnagar, E. Pippe, M. Alexe, and D. Hesse, Microstructure of highly strained $\mathrm{BiFeO}_{3}$ thin films: Transmission electron microscopy and electron-energy loss spectroscopy studies, J. Appl. Phys. 115, 043526 (2014).

[52] See Supplemental Material at http://link.aps.org/supplemental/ 10.1103/PhysRevMaterials.3.034410 for a description on how the initial configuration of the dislocation models was built, and the total density of states far from the dislocation core.
[53] R. P. Borges, W. Guichard, J. G. Lunney, J. M. D. Coey, and F. Ott, Magnetic and electric "dead" layers in $\left(\mathrm{La}_{0.7} \mathrm{Sr}_{0.3}\right) \mathrm{MnO}_{3}$ thin films, J. Appl. Phys. 89, 3868 (2001).

[54] P. Yu, W. Luo, D. Yi, J. X. Zhang, M. D. Rossell, C.-H. Yang, L. You, G. Singh-Bhalla, S. Y. Yang, Q. He, Q. M. Ramasse, R. Erni, L. W. Martin, Y. H. Chu, S. T. Pantelides, S. J. Pennycook, and R. Ramesh, Interface control of bulk ferroelectric polarization, Proc. Natl. Acad. Sci. 109, 9710 (2012).

[55] M. Watanabe, E. Okunishi, and K. Ishizuka, Analysis of spectrum-imaging datasets in atomic-resolution electron microscopy, Microsc. Anal. 23, 5 (2009).

[56] A. Tonomura, T. Matsuda, and J. Endo, Holographic interference electron microscopy for determining specimen magnetic structure and thickness distribution, Phys. Rev. B 34, 3397 (1986).

[57] N. T. Nelson, B. Winchester, Y. Zhang, S.-J. Kim, A. Melville, C. Adamo, C. M. Folkman, S.-H. Baek, C.-B. Eom, D. G. Schlom, L.-Q. Chen, and X. Pan, Spontaneous vortex nanodomain arrays at ferroelectric heterointerfaces, Nano Lett. 11, 828 (2011).

[58] M. J. Hÿtch, E. Snoeck, and R. Kilaas, Quantitative measurement of displacement and strain fields from HREM micrographs, Ultramicroscopy 74, 131 (1998).

[59] S. Plimpton, Fast parallel algorithms for short-range molecular-dynamics, J. Comput. Phys. 117, 1 (1995).

[60] V. Grillo and E. Rotunno, STEM_CELL: A software tool for electron microscopy: Part I-simulations, Ultramicroscopy 125, 97 (2013).

[61] J. J. Rehr, J. J. Kas, F. D. Vila, M. P. Prange, and K. Jorissen, Parameter-free calculations of x-ray spectra with FEFF9, Phys. Chem. Chem. Phys. 12, 5503 (2010).

[62] L. Hedin and S. Lundqvist, Effects of electron-electron and electron-phonon interactions on the one-electron states of solids, Solid State Phys. 23, 1 (1970). 